\title{
Free-Space Quantum Electrodynamics with a Single Rydberg Superatom
}

\author{
Asaf Paris-Mandoki, ${ }^{1,2, \dagger}$ Christoph Braun, ${ }^{1,2}$ Jan Kumlin, ${ }^{3}$ Christoph Tresp ${ }^{1,2}$ Ivan Mirgorodskiy, ${ }^{1}$ \\ Florian Christaller, ${ }^{1,2}$ Hans Peter Büchler, ${ }^{3}$ and Sebastian Hofferberth ${ }^{1,2, *}$ \\ ${ }^{1}$ Physikalisches Institut and Center for Integrated Quantum Science and Technology, \\ Universität Stuttgart, 70569 Stuttgart, Germany \\ ${ }^{2}$ Department of Physics, Chemistry and Pharmacy, University of Southern Denmark, 5230 Odense, Denmark \\ ${ }^{3}$ Institut für Theoretische Physik III and Center for Integrated Quantum Science and Technology, \\ Universität Stuttgart, 70569 Stuttgart, Germany
}

(Received 11 May 2017; revised manuscript received 14 July 2017; published 17 October 2017)

\begin{abstract}
The interaction of a single photon with an individual two-level system is the textbook example of quantum electrodynamics. Achieving strong coupling in this system has so far required confinement of the light field inside resonators or waveguides. Here, we demonstrate strong coherent coupling between a single Rydberg superatom, consisting of thousands of atoms behaving as a single two-level system because of the Rydberg blockade, and a propagating light pulse containing only a few photons. The strong lightmatter coupling, in combination with the direct access to the outgoing field, allows us to observe, for the first time, the effect of the interactions on the driving field at the single-photon level. We find that all our results are in quantitative agreement with the predictions of the theory of a single two-level system strongly coupled to a single quantized propagating light mode.
\end{abstract}

DOI: 10.1103/PhysRevX.7.041010

The interaction between a single emitter and individual photons is a fundamental process in nature [1], underlying many phenomena such as vision and photosynthesis as well as applications including imaging, spectroscopy, or optical information processing and communication. In the strongcoupling limit, where the coherent interaction between a single photon and an individual emitter exceeds all decoherence and loss rates, a single emitter can function as an interface between stationary and flying qubits, a central building block for future quantum networks $[2,3]$. Such a quantum optical node is able to mediate effective photon-photon interactions, thus enabling deterministic alloptical quantum gates [4-6].

One groundbreaking scheme to achieve strong coupling is the use of electromagnetic (EM) cavities, where the photons are trapped within the finite volume of a highfinesse resonator. The physics of these systems is captured by the seminal Jaynes-Cummings model [7], which has been experimentally realized and extensively studied in atomic cavity quantum electrodynamics (QED) [8] and, more recently, in circuit QED systems combining on-chip

\footnotetext{
*hofferberth@sdu.dk

Present address: Instituto de Física, Universidad Nacional Autónoma de México, Mexico City 04510 Mexico.

Published by the American Physical Society under the terms of the Creative Commons Attribution 4.0 International license. Further distribution of this work must maintain attribution to the author(s) and the published article's title, journal citation, and DOI.
}

Subject Areas: Atomic and Molecular Physics, Quantum Physics microwave resonators with superconducting two-level systems $[9,10]$. Achieving a strong interaction between a propagating photon and a single emitter opens the possibility to realize novel quantum-optical devices where atoms process photonic qubits on the fly and facilitate the preparation of nonclassical states of light [11]. However, mode matching between the input field and the dipolar emission pattern of the quantum emitter in free space is challenging and has so far limited the achievable coupling strength [12-14]. Waveguide QED systems seek to overcome this limitation by transversely confining the propagating EM mode coupled to one or more emitters [15-21].

Here, we report on the realization of coherent coupling between a propagating few-photon optical field and a single Rydberg superatom in free space. By exploiting the Rydberg blockade effect in an atomic ensemble [22-25], which allows, at most, a single excitation shared among all $N$ constituents, we turn about $10^{4}$ individual ultracold atoms into a single effective two-level quantum system. The collective nature of this excitation enhances the coupling of the light field to the superatom by a factor of $\sqrt{N}$ compared to the single-atom coupling strength and guarantees an enhanced directed emission in the forward direction $[22,26]$. The resulting large coupling enables us to drive Rabi oscillations of the single superatom with a fewphoton probe pulse and to observe, for the first time, the effects of the coherent emitter-photon interaction on the photon-photon correlations of the outgoing field. We show that our system is well described by the theory of a single quantum emitter strongly coupled to a one-dimensional quantized light mode and that the light-matter coupling we 
achieve in free space is competitive with state-of-the-art optical waveguide QED systems [18,20,21].

We implement our single Rydberg superatom by focusing a weak 780-nm probe field (beam waist $w_{\text {probe }}=6.5 \mu \mathrm{m}$ ), together with a strong counterpropagating control field at $480 \mathrm{~nm}$ (beam waist $w_{\text {control }}=14 \mu \mathrm{m}$ ) into an optically trapped ensemble of ultracold ${ }^{87} \mathrm{Rb}$ atoms $(T=6 \mu \mathrm{K})$ [Fig. 1(a) and Appendix A]. The few-photon coherent probe field, with a photon rate $\mathcal{R}_{\text {in }}$, couples the ground $|g\rangle=\left|5 S_{1 / 2}, F=2, m_{F}=2\right\rangle$ and intermediate $|e\rangle=\left|5 P_{3 / 2}, F=3, m_{F}=3\right\rangle$ states with a Rabi frequency $g_{0} \sqrt{\mathcal{R}_{\text {in }}}$, where $g_{0}$ is the single-atom-single-photon coupling constant, determined by the geometry of the setup. The control field provides coupling between $|e\rangle$ and the Rydberg state $|r\rangle=\left|111 S_{1 / 2}, m_{J}=1 / 2\right\rangle$ with Rabi frequency $\Omega=2 \pi \times 10 \mathrm{MHz}$ [Fig. 1(b)]. Using a large intermediate-state detuning $\Delta=2 \pi \times 100 \mathrm{MHz} \gg \Gamma_{e}, \Omega$, the intermediate state can be adiabatically eliminated. Setting the two-photon detuning $\delta$ to Raman resonance, the dynamics for each atom simplifies to those of a resonantly coupled two-level system between $|g\rangle$ and $|r\rangle$

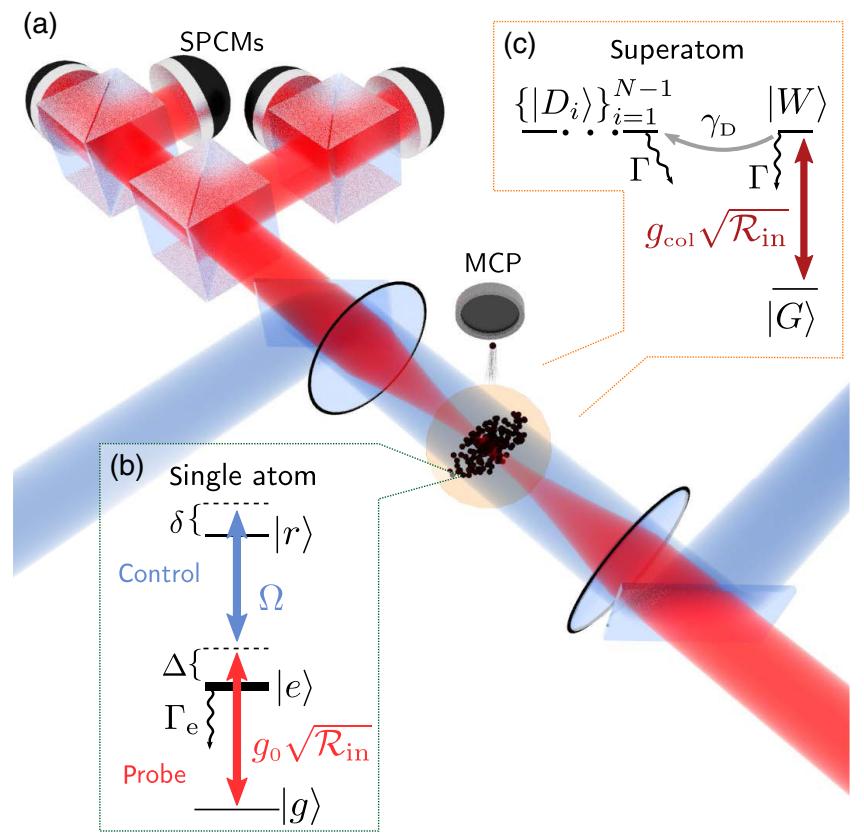

FIG. 1. Experimental setup and level scheme. (a) An ensemble of laser-cooled atoms is confined within a blockade volume using an optical dipole trap. Single-photon counting modules (SPCMs) are used to detect the light that interacts with the atoms, while a multichannel plate (MCP) detects the Rydberg atoms after ionization. (b) A few-photon probe field (red) and a strong control field (blue) couple the single-atom ground state $|g\rangle$ to a Rydberg state $|r\rangle$. Their respective Rabi frequencies are $g_{0} \sqrt{\mathcal{R}_{\text {in }}}$ and $\Omega$, where $g_{0}$ is the single-atom coupling constant for the probe field and $\mathcal{R}_{\text {in }}$ is the photon rate. (c) Because of the Rydberg blockade, the whole ensemble collectively behaves like a twolevel system with many-body states $|G\rangle$ and $|W\rangle$, including a loss channel to a set of collective dark states $\left\{\left|D_{i}\right\rangle\right\}_{i=1}^{N-1}$. with effective Rabi frequency $g_{0} \sqrt{\mathcal{R}_{\text {in }}} \Omega /(2 \Delta)$. The decay of $|r\rangle$ is dominated by spontaneous Raman decay via the $|e\rangle$ level with a rate $\Gamma=\Omega^{2} /(2 \Delta)^{2} \Gamma_{\mathrm{e}}$.

The interaction between Rydberg atoms results in a blockade volume inside which only a single excitation is allowed [22-25]. In our setup, both the transverse probe beam diameter and the longitudinal extent of the atomic cloud are smaller than the radius of the blockade volume, collectively coupling $N \approx 10^{4}$ atoms within this volume to the propagating light mode. Specifically, the $N$-body ground state $|G\rangle=\left|g_{1}, \ldots, g_{N}\right\rangle$ couples only to one manybody excited state $|W\rangle=(1 / \sqrt{N}) \sum_{j=1}^{N} e^{i \mathbf{k} \cdot \mathbf{x}_{j}}|j\rangle$, where $|j\rangle=\left|g_{1}, \ldots, r_{j}, \ldots, g_{N}\right\rangle$ is the state with the $j$ th atom in $|r\rangle$ and all others in $|g\rangle$, $\mathbf{k}$ is the sum of the wave vectors of the probe and control fields, and $\mathbf{x}_{j}$ denotes the position of the $j$ th atom. Ultimately, the ensemble of $N$ atoms acts as a single two-level superatom coupled to the probe light via the collective coupling constant $g_{\text {col }}=\sqrt{N} g_{0} \Omega /(2 \Delta)$ [Fig. 1(c)]. In addition to $|G\rangle$ and $|W\rangle$, the Hilbert space describing the system contains $N-1$ collective dark states $\left\{\left|D_{i}\right\rangle\right\}_{i=1}^{N-1}$ formed by linear combinations of $\{|j\rangle\}_{j=1}^{N}$. While these states still contain an excitation that blocks the medium, they are not coupled to the probe light. The exchange of virtual photons between atoms has been shown to provide coupling between the $|W\rangle$ state and the collective dark states [27-30], which can alter the decay rate of the bright state [31,32]. Additionally, inhomogeneous dephasing acting on individual atoms can irreversibly drive the ensemble from $|W\rangle$ into the manifold of dark states $\left\{\left|D_{i}\right\rangle\right\}_{i=1}^{N-1}$, which enables the system to function as a single-photon absorber [33,34].

To observe the coherent dynamics of the superatom, we send a Tukey-shaped probe pulse with a peak photon rate $\mathcal{R}_{\text {in }}$ into the atomic cloud. After its interaction with the ensemble, the probe light is collected by four single-photon counters [Fig. 1(a)]. Alternatively, the Rydberg population in the ensemble at any time is measured by counting the ions produced by a fast field-ionization pulse with a microchannel plate detector (see Appendix B). In Figs. 2(a) and 2(b), we show average photon time traces and ion signals for $\mathcal{R}_{\text {in }}=12.4 \mu \mathrm{s}^{-1}$ and $\mathcal{R}_{\text {in }}=2.6 \mu \mathrm{s}^{-1}$. First, we observe the collectively enhanced Rabi oscillation of the Rydberg population [23-25,35-37]. Importantly, the number of Rydberg atoms throughout the whole pulse stays below one, showing that our ensemble is indeed fully blockaded and well described as a single superatom. The coherent dynamics of the system also causes a periodic modulation of the outgoing photon rate $\mathcal{R}_{\text {out }}$. Figure 2(c) shows this modulation $\Delta \mathcal{R}(t)=\mathcal{R}_{\text {in }}(t)-\mathcal{R}_{\text {out }}(t)$ for a range of input rates, down to $\mathcal{R}_{\text {in }}=1.5 \mu \mathrm{s}^{-1}$, which corresponds to a mean number of photons $\bar{N}_{\mathrm{ph}}=9$ in the probe pulse.

In order to quantitatively describe our results, we consider the Hamiltonian of a single two-level system coupled to a quantized light field 


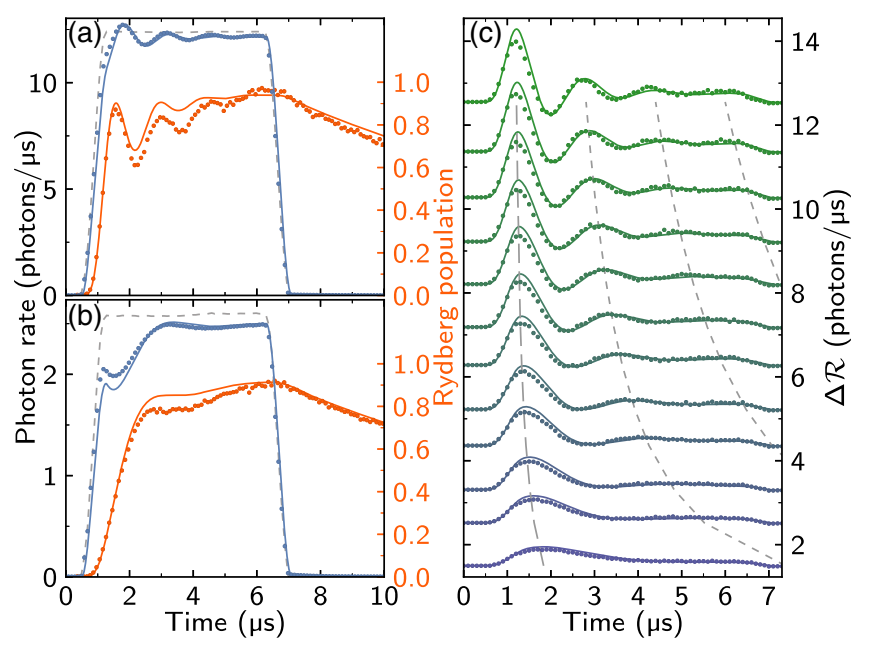

FIG. 2. Time evolution of the photon signal and Rydberg population. $(a, b)$ Time traces of the outgoing probe photon rate (blue points) and Rydberg population (orange points) for input pulses (dashed gray line) with peak photon rates $\mathcal{R}_{\text {in }}=12.4 \mu \mathrm{s}^{-1}$ (a) and $\mathcal{R}_{\text {in }}=2.6 \mu \mathrm{s}^{-1}$ (b), corresponding to a mean number $\bar{N}_{\mathrm{ph}}$ of 71.6 and 15.1 photons in the pulse. The Rabi oscillation of the single superatom is visible both in the excited-state population and in the modulation of the transmitted probe light. Solid lines are fits to the data using our model. (c) Difference signal $\Delta \mathcal{R}(t)$ between the incoming and outgoing pulses (dots) for different input photon rates $\mathcal{R}_{\text {in }}$. Each data set is vertically shifted by the corresponding $\mathcal{R}_{\text {in }}$. Solid lines are again the result of fitting the full data set with our theory model using a single set of fit parameters. Dashed lines indicate the expected positions of the Rabi oscillation peaks based on the fitted parameters, showing the scaling of the Rabi period with $1 / \sqrt{\bar{N}_{\text {ph }}}$. Error bars in (a)-(c) are SEM and are smaller than the data points.

$H=\int \frac{d k}{2 \pi} \hbar c k a_{k}^{\dagger} a_{k}+\frac{\hbar g_{\mathrm{col}}}{2}\left(E^{\dagger}(0) \sigma_{G W}+E(0) \sigma_{G W}^{\dagger}\right)$,

where $a_{k}$ and $a_{k}^{\dagger}$ are photon annihilation and creation operators, $E(x)=[\sqrt{c} /(2 \pi)] \int e^{i k x} a_{k} d k$ is the electric-field operator measured in $\sqrt{\text { photons/time }}$, and $\sigma_{\alpha \beta}=|\alpha\rangle\langle\beta|$. Since the probe photons irreversibly leave after a single pass through the system, we can solve and trace out the time dependence of the photonic part (see Appendix D). For a coherent input state, we obtain a master equation for the atomic density matrix $[11,38]$

$$
\begin{aligned}
\partial_{t} \rho(t)= & -\frac{i}{\hbar}\left[H_{0}(t), \rho(t)\right]+(\kappa+\Gamma) \mathcal{L}\left[\sigma_{G W}\right] \rho(t) \\
& +\gamma_{D} \mathcal{L}\left[\sigma_{D W}\right] \rho(t)+\Gamma \mathcal{L}\left[\sigma_{G D}\right] \rho(t),
\end{aligned}
$$

where $\mathcal{L}[\sigma] \rho=\sigma \rho \sigma^{\dagger}-\left(\sigma^{\dagger} \sigma \rho+\rho \sigma^{\dagger} \sigma\right) / 2$ is the Lindblad superoperator and the effective Hamiltonian is

$$
H_{0}(t)=\hbar \sqrt{\kappa}\left(\alpha^{*}(t) \sigma_{G W}+\alpha(t) \sigma_{G W}^{\dagger}\right)
$$

with the coherent field amplitude $\alpha(t)$ related to the timedependent mean photon rate by $|\alpha(t)|^{2}=\mathcal{R}_{\text {in }}(t)$ and the rate of emission $\kappa=g_{\mathrm{col}}^{2} / 4$ of the two-level system into the strongly coupled mode. In addition to this intrinsic decay channel, which is derived from the Hamiltonian in Eq. (1), we phenomenologically add the spontaneous Rydberg atom decay rate $\Gamma$ of the excited state and the dephasing rate $\gamma_{D}$ of the superatom state $|W\rangle$ into the manifold of dark states $|D\rangle$. The Rydberg population is then given by $\rho_{W W}+\rho_{D D}$, while the outgoing electric field is

$$
E(t)=\alpha(t)-i \sqrt{\kappa} \sigma_{G W}(t) .
$$

The equal-time expectation values for the electric-field operator therefore reduce to the determination of equaltime correlations in $\sigma_{G W}^{\dagger}(t)$, which are obtained by the numerical solution of Eq. (2). In particular, the expectation value of the photon flux at retarded time $s=t-x / c$ is given by

$$
\begin{aligned}
\left\langle E^{\dagger}(s) E(s)\right\rangle= & |\alpha(s)|^{2}+\kappa\left\langle\sigma_{G W}^{\dagger}(s) \sigma_{G W}(s)\right\rangle \\
& -i \sqrt{\kappa}\left[\alpha^{*}(s)\left\langle\sigma_{G W}(s)\right\rangle-\alpha(s)\left\langle\sigma_{G W}^{\dagger}(s)\right\rangle\right] .
\end{aligned}
$$

The solid lines in Figs. 2(a)-2(c) are the result of fitting the above model to the respective sets of time traces. For the set in Figs. 2(a) and 2(b), we obtain the values $\kappa=0.428 \mu \mathrm{s}^{-1}$, $\Gamma=0.069 \mu \mathrm{s}^{-1}$, and $\gamma_{D}=1.397 \mu \mathrm{s}^{-1}$.

For a single atom in free space, the coupling with a photon, quantified by $\kappa$, can maximally become as large as the spontaneous decay rate of the bare atom $\Gamma$ in the case of perfect mode matching [39]. In the superatom case, the coupling $g_{\text {col }} \sim \sqrt{\kappa}$ and the decay $\kappa$ into a specific mode can be boosted solely through the collective enhancement of the atom-light interaction, without any confinement of the propagating light. As a consequence, the superatom spontaneously emits with probability $\beta=\kappa /(\kappa+\Gamma)=0.86$ into the forward direction of the strongly coupled mode, while loss of photons due to scattering out of the propagating mode with rate $\Gamma$ is minimal (see Appendix F). The main decoherence source in our current implementation is the superatom dephasing $\gamma_{D}$; we expect that thermal motion of the individual atoms in the superatom are the leading mechanism for this dephasing, which could be significantly reduced by technical improvement of our setup [40,41]. However, a coherent virtual exchange of photons can provide an additional coherent dynamics for the superatom [27-30]. Given the excellent agreement between the experimental data and our model, we conclude that, in the present experimental regime, the potential influence of this coherent term is well accounted for by the phenomenological dephasing rate $\gamma_{D}$.

In contrast to cavity QED, in free-space and waveguide QED, an increase of the coupling $g_{\mathrm{col}}$ necessarily increases the decay rate $\kappa$, resulting in an intrinsic damping of these 
systems, preventing perfect transfer of a photonic qubit to a matter qubit within a finite time $[39,42,43]$. To further illustrate this point, Fig. 3 shows the visibility of Rabi oscillations of an ideal $\left(\Gamma=\gamma_{D}=0\right)$ two-level atom (see Appendix E) as a function of the dimensionless coupling parameter $\lambda=\kappa \tau$, where $\tau$ is the length of the incoming pulse, and the mean photon number in the pulse $\bar{N}_{\text {ph }}=\mathcal{R}_{\text {in }} \tau$. For $\lambda \gg 1$, the atom decays very quickly, and photons exhibit correlations only on a time scale $1 / \kappa$. This results in an overdamped regime, where the system settles to a nonzero probability to find the superatom in the excited state without undergoing any Rabi oscillations. In the opposite limit $\lambda \ll 1$, a minimum number of photons is required to drive a $\pi$ pulse, which defines a crossover (dashed line in Fig. 3) between the regime with Rabi oscillations and the weak driving regime, where the excitation probability remains below its steady-state value during the pulse duration $\tau$. For our current experiments, $\lambda=2.2$ (see Appendix C), thus placing our setup close to the ideal regime where multiple Rabi cycles are observable within the decay time $1 / \kappa$ for very low $\bar{N}_{\text {ph }}$.

Since we have access to the full counting statistics of the outgoing light, we can investigate how the dynamics of the

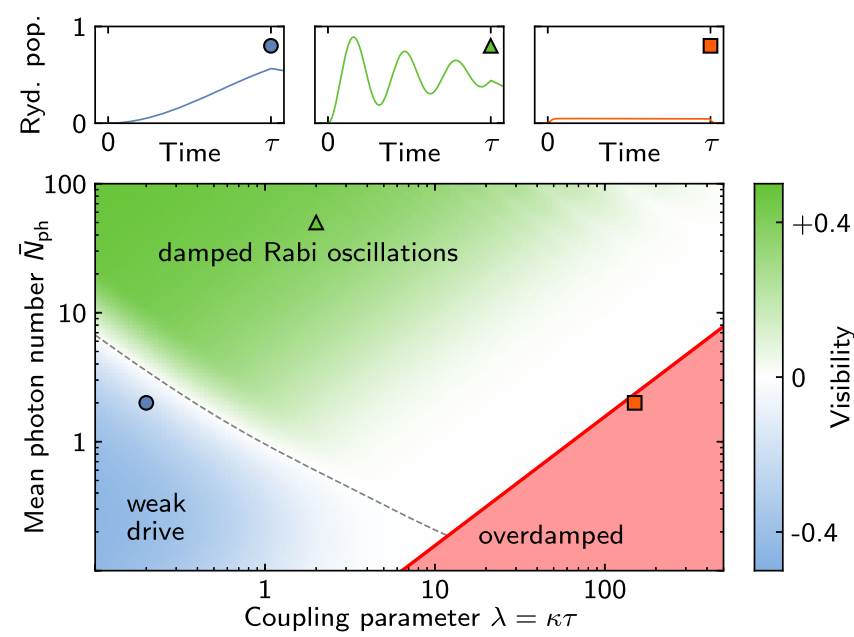

FIG. 3. Dynamical phase diagram of a driven atom in free space. (Bottom panel) The diagram shows the visibility of Rabi oscillations, defined as $\max _{0 \leq t \leq \tau}\left[\rho_{W W}(t)\right]-\rho_{W W}(t=\infty)$, of an ideal $\left(\Gamma=\gamma_{D}=0\right)$ atom driven by a propagating field. In contrast to cavity QED, the coupling and the decay of photons from the system are not independent in free-space and waveguide QED. For large coupling to the propagating mode $(\lambda=\kappa \tau \gg 1)$, the enhanced emission into this mode results in an overdamped system, where the number of photons required to observe Rabi oscillations increases with coupling strength. For $\lambda \ll 1$, a large number of photons is required to drive the system with a $\pi$ pulse, defining a crossover (dashed line) between the regime of damped Rabi oscillations and the weak driving regime at lower mean photon number. For our experiment, we find $\lambda=2.2$. (Top panels) Examples of the variation of the Rydberg population with time for the points indicated in the main diagram. coupled system results in correlations between emerging probe photons. We show in Figs. 4(a) and 4(b) the measured two-time correlation functions $g^{(2)}\left(t_{1}, t_{2}\right)$ for $\mathcal{R}_{\text {in }}=12.4 \mu \mathrm{s}^{-1}$ and $\mathcal{R}_{\text {in }}=2.5 \mu \mathrm{s}^{-1}$. The periodic structure of bunching and antibunching can be understood as the rearrangement of photons in the coherent input beam due to the absorption and stimulated emission by the superatom, emphasizing the long coherence time of the superatomphoton interaction. Figures 4(c) and 4(d) show the corresponding calculated correlations from our model. The evaluation of the two-point correlation function, in contrast to the intensity expectation value, requires the determination of correlations of the operators $\sigma_{G W}^{\dagger}(t)$ at different times, i.e.,

$$
g^{(2)}\left(s_{1}, s_{2}\right)=\frac{\left\langle E^{\dagger}\left(s_{1}\right) E^{\dagger}\left(s_{2}\right) E\left(s_{2}\right) E\left(s_{1}\right)\right\rangle}{\left\langle E^{\dagger}\left(s_{1}\right) E\left(s_{1}\right)\right\rangle\left\langle E^{\dagger}\left(s_{2}\right) E\left(s_{2}\right)\right\rangle} .
$$

It is a remarkable property of a single atom coupled to a single photonic mode that these expectation values can be determined by the quantum regression theorem without involving any additional approximations. While, in general, the quantum regression theorem relies on a Born approximation quenching the correlations between the bath and the system [44], here, the emitted photons never interact with the superatom again, which is exactly the requirement for the validity of the quantum regression theorem [38]. To verify this statement, we use the exact diagonalization of the Hamiltonian in Eq. (1) by means of the Bethe ansatz [45-47] to obtain the wave function of the outgoing pulse for few-photon Fock states. We then find perfect agreement for the correlation function derived from the exact wave function via the Bethe ansatz and the above derivation using the quantum regression theorem for a coherent state with low mean photon number. Furthermore, the theory results are in excellent agreement with the experimental data, including the correlations beyond the duration of the pulse. These originate from collective spontaneous emission of single photons after the input pulse has left the sample, which can only occur if the superatom is in state $|W\rangle$ at the end of the driving pulse. The observed correlations indicate that, because of the effective photonphoton interaction mediated by the single superatom, photons separated by up to $5 \mu \mathrm{s}$ in time become entangled [48]. To illustrate this point, consider two incoming photons: The first photon passing the atom results in a superposition state of the photon either being absorbed and the superatom excited or the photon having passed the atom without exciting it [28]. The second photon passing by then has restricted options depending on whether the superatom state is already occupied or not; i.e., it can only be absorbed if the first photon was not, resulting in spatial entanglement between the two photons, mediated by their subsequent interaction with a single two-level system. 


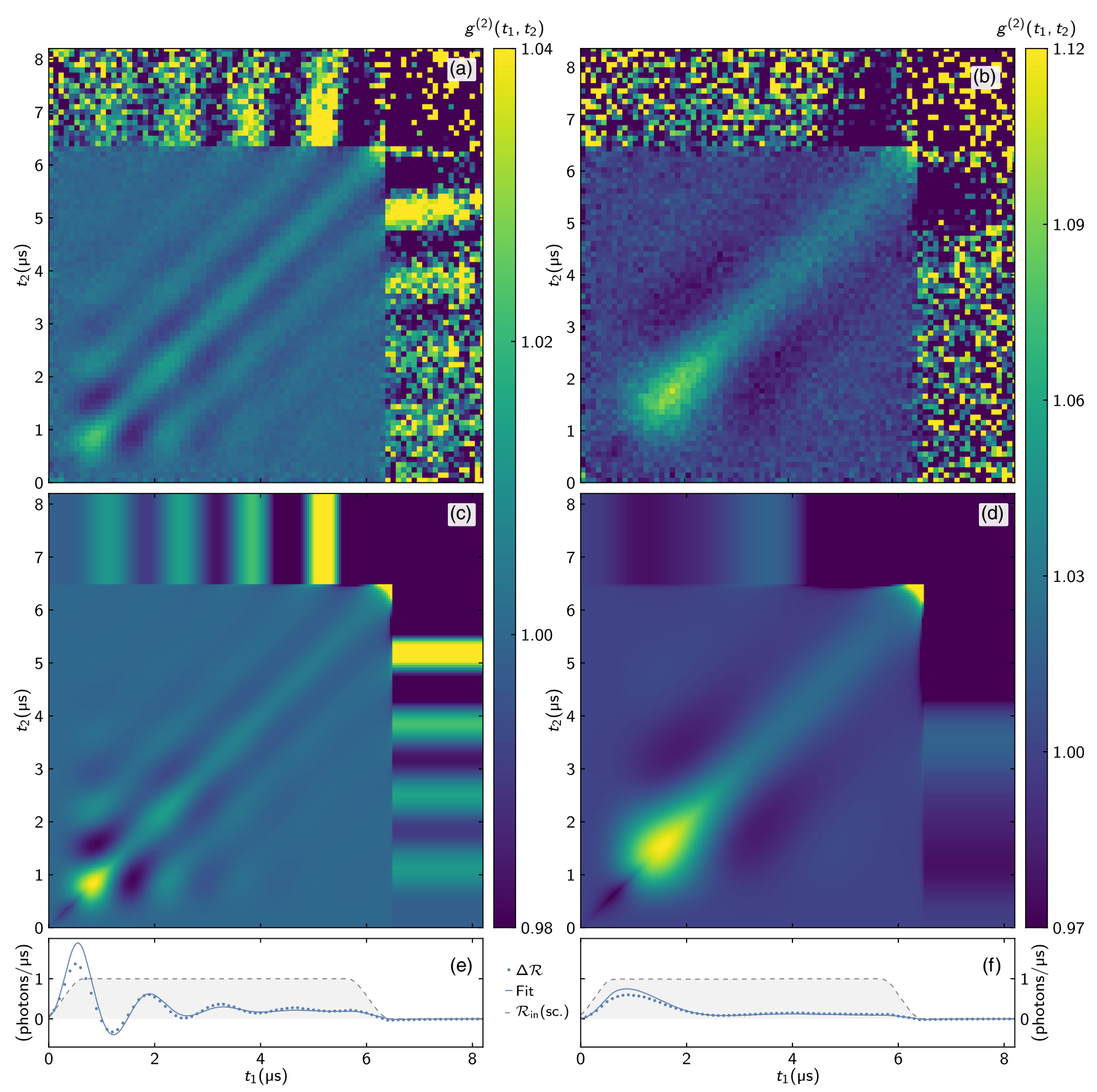

FIG. 4. Correlations of the outgoing probe field. (a,b) Measured two-time correlations $g^{(2)}\left(t_{1}, t_{2}\right)$ for pulses with $\mathcal{R}_{\text {in }}=12.4 \mu \mathrm{s}^{-1}$ and $\mathcal{R}_{\text {in }}=2.5 \mu \mathrm{s}^{-1}$ corresponding to the time traces in Figs. 2(a) and 2(b). (c,d) Corresponding calculated correlation functions using the values of $\kappa, \Gamma$, and $\gamma_{D}$ obtained by fitting the time traces in Fig. 2(a) and 2(b). (e,f) Measured (dotted line) and simulated (solid line) $\Delta \mathcal{R}_{\text {in }}$ together with scaled input pulses (dashed gray) for reference.

In conclusion, our measurements demonstrate the realization of strong light-matter coupling in free space, through collective enhancement of the coupling strength without any confining structures for the propagating light mode. The tunable dephasing of the superatom into dark states creates additional functionality beyond the conventional two-level system [33,34]. The scaling of our system to complex arrangements of multiple superatoms is straightforward and paves the way towards quantum optical networks [2,3] and the realization of strongly correlated states of light and matter [6]. The directionality of the superatom emission can be used to implement a cascaded quantum system for dissipative entanglement generation among the superatoms $[49,50]$. 
We thank Ofer Firstenberg and Jürgen Eschner for stimulating discussions and Hannes Gorniaczyk for contributions to the experiment. This work is funded by the German Research Foundation (Emmy-Noether-Grant No. HO 4787/1-1, GiRyd Project No. HO 4787/1-3, and SFB/TRR21 Project No. C12), the Ministry of Science, Research and the Arts of Baden-Württemberg (RiSC Grant No. 33-7533.-30-10/37/1), European Union under the ERC Consolidator Grant SIRPOL (Grant No. 681208), and in part by the National Science Foundation under Grant No. NSF PHY-1125915.

A. P.-M. and C. B. contributed equally to this work.

\section{APPENDIX A: PREPARATION OF A SINGLE SUPERATOM}

To prepare the ultracold atomic ensemble that forms our single Rydberg superatom, we initially load ${ }^{87} \mathrm{Rb}$ atoms into a magneto-optical trap (MOT) from $10^{-10}$ mbar rubidium background pressure in an ultrahigh vacuum chamber, resulting in $5 \times 10^{6}$ laser-cooled atoms after $1 \mathrm{~s}$ of loading. After compressing the MOT by increasing the gradient of the quadrupole magnetic field, the atoms are loaded into a dipole trap formed by two crossed 1070-nm beams, intersecting under an angle of $31.4^{\circ}$, and an additional elliptic dimple beam at $855 \mathrm{~nm}$ perpendicular to the long axis of the original trap. Subsequently, atoms are further cooled by forced evaporation for $700 \mathrm{~ms}$ by reducing the power of the two 1070-nm dipole trap beams. During evaporative cooling, the cloud is additionally cooled during two stages of Raman sideband cooling. The stages are $10 \mathrm{~ms}$ long and occur after $89 \mathrm{~ms}$ and $589 \mathrm{~ms}$ of evaporation, eventually reaching a final cloud temperature of $6 \mu \mathrm{K}$. The final atomic cloud contains 25000 atoms in a pancake-shaped harmonic trap, with a Gaussian density profile with widths $\sigma_{z}=6 \mu \mathrm{m}$ and $\sigma_{r}=10 \mu \mathrm{m}$. The Rydberg state $111 S_{1 / 2}$ was chosen such that the longitudinal diameter of the cloud, as well as the transverse diameter of the probe beam, are significantly smaller than the minimum Rydberg blockade radius $r_{b}=25.5 \mu \mathrm{m}$ at the highest photon rate $\mathcal{R}_{\text {in }}=12.4 \mu \mathrm{s}^{-1}$, resulting in a fully blockaded ensemble of atoms coupled to the probe (waist $\left.w_{\text {probe }}=6.5 \mu \mathrm{m}\right)$ and control light (waist $\left.w_{\text {control }}=14 \mu \mathrm{m}\right)$.

\section{APPENDIX B: PULSED FEW-PHOTON EXPERIMENTS}

After preparation of the atomic sample, 1000 individual experiments as described in the main text are performed within a time of $100 \mathrm{~ms}$. For each experiment, the optical trapping beams are turned off for $14 \mu \mathrm{s}$, with the atoms being recaptured after each pulse sequence. The Tukeyshaped probe pulses have rise and fall times of $0.8 \mu \mathrm{s}$ and an uptime of $5 \mu \mathrm{s}$. After a $2-\mu$ s initial wait time to fully turn off the crossed dipole trap, the control light is turned on
$2 \mu$ s before the probe pulse and remains on during the remaining $12 \mu \mathrm{s}$. Alternatively, for measuring the Rydberg population, a field ionization pulse is applied at a varying time, and the produced ions are detected on a multichannel plate (MCP). An electric-field amplitude $F>85 \mathrm{~V} \mathrm{~cm}^{-1}$ ensures that all Rydberg atoms are ionized. After 1000 individual experiments, the atomic cloud is released, and after a 10 -ms wait time, we again perform 1000 experiments without an atomic cloud for reference. Because of the finite detuning from the intermediate state, the probe transmission in the absence of the control field is $99 \%$. We detect a photon after it passes through the atomic ensemble with an overall detection efficiency of $29 \%$; after field ionizing, an individual Rydberg atom is detected by the MCP with an overall detection efficiency of $30 \%$.

\section{APPENDIX C: DATA ANALYSIS AND STATISTICS}

Each data point of the photon time traces in Figs. 2(a) and 2(b) corresponds to an average of $36.85 \times 10^{6}$ and $21.73 \times 10^{6}$ individual measurements, respectively. From these data sets, we also obtain the correlation functions in Figs. 4(a) and 4(b). Each data point of the Rydberg population measurements in Figs. 2(a) and 2(b) represents an average over $32 \times 10^{3}$ and $61 \times 10^{3}$ experiments. As stated in the main text, we fit our numerical model to the experimental data and obtain a common set of parameters for Figs. 2(a) and 2(b), namely, $\kappa=0.428 \mu \mathrm{s}^{-1}$, $\Gamma=0.069 \mu \mathrm{s}^{-1}$, and $\gamma_{D}=1.397 \mu \mathrm{s}^{-1}$. These parameters are used for the calculated correlation functions in Figs. 4(c) and 4(d). Each data point in the photon traces in Fig. 2(c) represents the average of $1.86 \times 10^{6}$ experiments. Here, we fit with a single set of parameters to all shown traces simultaneously, obtaining $\kappa=0.322 \mu \mathrm{s}^{-1}$, $\Gamma=0.069 \mu \mathrm{s}^{-1}$, and $\gamma_{D}=1.326 \mu \mathrm{s}^{-1}$. The differences in $\kappa$ and $\gamma_{D}$ between the two data sets stem from slightly different numbers of atoms $N$ in the superatom for the two experiment runs. The single-atom decay $\Gamma$ is set solely by the control laser parameters and Rydberg lifetime and thus does not change between runs. For all data shown, the standard error of the mean (SEM) is smaller than the size of the displayed dots. For the calculation of the dimensionless coupling parameter $\lambda=\kappa \tau$, we use the mean of the two measured $\kappa$ and an effective length of the probe pulse of $\tau=5.8 \mu$ s.

\section{APPENDIX D: DERIVATION OF MASTER EQUATION}

We start with the Hamiltonian Eq. (1), which describes the coherent coupling of the superatom to the optical mode of the incoming laser field. The derivation of the master equation closely follows the methods described in standard textbooks [51] and recent publications on atom-light coupling in one-dimensional waveguides $[11,38]$. The first 
step is to derive the Heisenberg equation of motion for the photonic field operators

$\partial_{t} a_{k}(t)=-\frac{i}{\hbar}\left[a_{k}, H\right]=-i c k a_{k}(t)-i \sqrt{\kappa c} \sigma_{G W}(t)$,

with $\sigma_{G W}=|G\rangle\langle W|$ and the coupling strength $\sqrt{\kappa}=g_{\mathrm{col}} / 2$. This equation has a simple solution that leads to a connection between the outgoing electric field and the operator $\sigma_{G W}$, which describes the coherences in the superatom,

$$
\begin{aligned}
a_{k}(t)= & e^{-i c k\left(t-t_{0}\right)} a_{k}\left(t_{0}\right) \\
& -i \sqrt{\kappa c} e^{-i c k\left(t-t_{0}\right)} \int_{t_{0}}^{t} d s e^{i c k\left(s-t_{0}\right)} \sigma_{G W}(s) .
\end{aligned}
$$

Here, $t_{0}$ denotes the initial time, with the condition that the incoming photon field has not yet reached the superatom. Without loss of generality, we set $t_{0}=0$. Then, the electricfield operator reduces to

$$
\begin{aligned}
E(x, t) & =\bar{E}(c t-x)-i \sqrt{\kappa} c \int_{0}^{t} d s \int \frac{d k}{2 \pi} e^{-i c k(t-s)+i k x} \sigma_{G W}(s), \\
& =\bar{E}(c t-x)-i \sqrt{\kappa} \sigma_{G W}(t-x / c) \theta(x) \theta(c t-x) .
\end{aligned}
$$

Here, $\bar{E}$ denotes the noninteracting electric-field operator, and $\theta(x)$ is the Heaviside function with the definition $\theta(0)=1 / 2$. For an incoming coherent state, the noninteracting electric field $\bar{E}(c t)$ can be replaced by the amplitude of the coherent field $\alpha(t) \equiv\langle\bar{E}(c t)\rangle$, which characterizes the incoming photon rate by $|\alpha(t)|^{2}=\mathcal{R}_{\text {in. }}$. Alternatively, it would be possible to apply the wellestablished Mollow transformation [52], leading to the same final result for the master equation. For an arbitrary operator $A$ acting on the superatom alone, its Heisenberg equation of motion reduces to

$$
\begin{aligned}
\partial_{t} A(t)= & -i \sqrt{\kappa}\left[A(t), \sigma_{G W}^{\dagger}(t)\right] \alpha(t)-\frac{\kappa}{2}\left[A(t), \sigma_{G W}^{\dagger}(t)\right] \sigma_{G W}(t) \\
& -i \sqrt{\kappa} \alpha^{*}(t)\left[A(t), \sigma_{G W}(t)\right]+\frac{\kappa}{2} \sigma_{G W}^{\dagger}(t)\left[A(t), \sigma_{G W}(t)\right] .
\end{aligned}
$$

The right-hand side can be split into a coherent part given by

$$
\begin{aligned}
- & i \sqrt{\kappa}\left[A(t), \sigma_{G W}^{\dagger}(t)\right] \alpha(t)-i \sqrt{\kappa} \alpha^{*}(t)\left[A(t), \sigma_{G W}(t)\right] \\
& =-\frac{i}{\hbar}\left[A(t), H_{0}(t)\right]
\end{aligned}
$$

with the Hamiltonian $H_{0}(t)$ in Eq. (3), while the remaining terms describe the spontaneous emission into the photonic mode.
Using the relation, $\partial_{t}\langle A\rangle=\operatorname{Tr}\left\{A \partial_{t} \rho(t)\right\}$, with $\rho(t)$ the reduced density matrix for the atomic system alone, the dissipative part reduces to the well-established Lindblad form

$\kappa \mathcal{L}\left[\sigma_{G W}\right] \rho(t)=\kappa\left(\sigma_{G W} \rho(t) \sigma_{G W}^{\dagger}(t)-\frac{1}{2}\left\{\sigma_{G W}^{\dagger} \sigma_{G W}(t), \rho(t)\right\}\right)$.

This term describes the enhanced spontaneous emission into the forward direction due to the collective character of the superatom. In addition, the superatom can also decay into transverse photonic modes, which is still determined by the standard single atom spontaneous emission rate $\Gamma$ (see Appendix F). Finally, we can add the dephasing into the dark states $\left\{\left|D_{i}\right\rangle\right\}_{i=1}^{N-1}$ as well as the decay by spontaneous emission of these dark states. The analysis is independent of the specific dark state the system dephases into, and therefore, we can account for the dephasing by losses into a single dark state $|D\rangle$ with a phenomenological dephasing rate $\gamma_{D}$. The microscopic mechanisms for the dephasing are, on one hand, Doppler shifts of the atoms, as well as inhomogeneous shifts of the Rydberg state level, and residual interactions between the individual atoms by resonant exchange interactions [11,27-29,53].

\section{APPENDIX E: ANALYTICAL SOLUTION OF THE MASTER EQUATION}

In the ideal case with $\Gamma=\gamma_{D}=0$, the master equation in Eq. (2) allows for an analytical solution for a driving field $\alpha=\sqrt{\mathcal{R}_{\text {in }}}$ switched on at time $t=0$. The full solution for the probability to be in the excited state $\rho_{W W}$ is given by

$\rho_{W W}(t)=\frac{4 \alpha^{2} \kappa}{\kappa^{2}+8 \alpha^{2} \kappa}\left(1-\left(\cos \Omega_{\mathrm{eff}} t+\frac{3 \kappa}{4 \Omega_{\mathrm{eff}}} \sin \Omega_{\mathrm{eff}} t\right) e^{-\frac{3}{4} \kappa t}\right)$,

with the effective Rabi frequency $\Omega_{\text {eff }}=\sqrt{4 \kappa \alpha^{2}-(\kappa / 4)^{2}}$. Introducing the dimensionless coupling parameter $\lambda=\kappa \tau$, where $\tau$ is the length of the incoming pulse, and the mean photon number in the pulse $\bar{N}_{\mathrm{ph}}=|\alpha|^{2} \tau$, the behavior of the superatom in free space can be described by a dynamical phase diagram, Fig. 3. First, it is important to stress that, in contrast to cavity QED, the coupling and the decay in this free-space setup are not independent. This implies that there is no "strong-coupling" regime as in cavity QED, where the coupling can be increased without affecting the spontaneous emission of the atom. Indeed, for increasing coupling strength $\kappa$ at fixed mean photon number $\bar{N}_{\text {ph }}$, the dissipation by the spontaneous emission increases, which reduces the visibility of the Rabi oscillations and eventually leads to the overdamped regime at $\bar{N}_{\mathrm{ph}}=\lambda / 64$. This overdamped regime is characterized by an imaginary 
effective Rabi frequency $\Omega_{\text {eff }}$; the red line in Fig. 3 shows this transition. In turn, for weak coupling $\lambda \ll 1$, we need to have a large number of photons in order to drive the system with a $\pi$ pulse, which defines a crossover between a regime with Rabi oscillations and the weak driving regime at lower mean photon number. This crossover is illustrated by the dashed line in Fig. 3.

In the experimentally relevant case of $\Gamma, \gamma_{D}>0$, the resulting master equation includes the additional level $|D\rangle$. This extended model can be solved to obtain the effective Rabi frequency $\Omega_{\text {eff }}\left(\kappa, \Gamma, \gamma_{D}\right)$. This solution is used to plot the lines predicting the Rabi oscillation maxima in Fig. 2(c).

\section{APPENDIX F: COLLECTIVE COUPLING AND DECAY INTO THE FORWARD-PROPAGATING MODE}

We start with a microscopic setup as realized in the experiment: A large number of atoms are localized within a harmonic trap. The density distribution of the ground-state atoms is given by a Gaussian profile with widths $\sigma_{z}$ along the direction of the incoming light field and $\sigma_{r}$ in the transverse direction with peak density $n_{0}$. Each atom is well described by a two-level atom with the ground state $|g\rangle$ and the excited Rydberg state $|r\rangle$, with the optical transition frequency $\omega=2 \pi c / \lambda$ and wavelength $\lambda$. In the following, we describe the two states of the atoms by the field operators $\psi_{g}^{\dagger}(\mathbf{r})$ for the ground state and $\psi_{r}^{\dagger}(\mathbf{r})$ for the Rydberg state, respectively. In terms of these operators, the ground-state density is defined as the expectation value

$$
n(\mathbf{r})=\left\langle\psi_{g}^{\dagger}(\mathbf{r}) \psi_{g}(\mathbf{r})\right\rangle=n_{0} e^{-z^{2} / 2 \sigma_{z}^{2}} e^{-\left(x^{2}+y^{2}\right) / 2 \sigma_{r}^{2}} .
$$

It is important to stress that for a thermal gas above quantum degeneracy, the statistics of the operator $\psi_{g}^{\dagger}(\mathbf{r})$ is irrelevant, but the correlations exhibit the fundamental property

$$
\begin{aligned}
& \left\langle\psi_{g}^{\dagger}(\mathbf{r}) \psi_{g}(\mathbf{r}) \psi_{g}^{\dagger}\left(\mathbf{r}^{\prime}\right) \psi_{g}\left(\mathbf{r}^{\prime}\right)\right\rangle \\
& \quad=g^{(2)}\left(\mathbf{r}, \mathbf{r}^{\prime}\right) n(\mathbf{r}) n\left(\mathbf{r}^{\prime}\right)+n(\mathbf{r}) \delta\left(\mathbf{r}-\mathbf{r}^{\prime}\right),
\end{aligned}
$$

with $g^{(2)}\left(\mathbf{r}, \mathbf{r}^{\prime}\right)$ the two-body correlation function. In the present system, the atoms are randomly distributed within the trap, and no correlations appear on length scales comparable to the wavelength $\lambda$, i.e., $g_{2}=1$. Next, we introduce the operators $S^{+}(\mathbf{r})=\psi_{r}^{\dagger}(\mathbf{r}) \psi_{g}(\mathbf{r})$, creating a Rydberg excitation from the ground state and $S^{-}(\mathbf{r})=$ $\psi_{g}^{\dagger}(\mathbf{r}) \psi_{r}(\mathbf{r})$ for a transition from the Rydberg state into the ground state. These operators satisfy the commutation relation

$$
\begin{aligned}
{\left[S^{-}(\mathbf{r}), S^{+}\left(\mathbf{r}^{\prime}\right)\right]=} & \psi_{g}^{\dagger}(\mathbf{r}) \psi_{g}(\mathbf{r}) \delta\left(\mathbf{r}-\mathbf{r}^{\prime}\right) \\
& -\psi_{r}^{\dagger}(\mathbf{r}) \psi_{r}(\mathbf{r}) \delta\left(\mathbf{r}-\mathbf{r}^{\prime}\right) .
\end{aligned}
$$

Then, the Hamiltonian describing the coupling between light and atomic ensemble within the rotating frame and using the rotating wave approximation reduces to

$H=\int \frac{d \mathbf{q}}{(2 \pi)^{3}} \hbar \omega_{\mathbf{q}} a_{\mathbf{q}}^{\dagger} a_{\mathbf{q}}+g \int d \mathbf{r}\left[S^{-}(\mathbf{r}) \mathcal{E}^{\dagger}(\mathbf{r})+\mathcal{E}(\mathbf{r}) S^{+}(\mathbf{r})\right]$,

with $g$ the dipole matrix element for the optical transition. In the following, we assume a polarization $\mathbf{p}$ of the dipole transition along the $x$ direction. Therefore, the electric-field operator in three dimensions takes the form

$$
\mathcal{E}(\mathbf{r})=\sum_{\mu} \int \frac{d \mathbf{q}}{(2 \pi)^{3}} c_{\mathbf{q}}^{\mu} a_{\mathbf{q}} e^{i \mathbf{q r}},
$$

with $c_{\mathbf{q}}^{\mu}=i \sqrt{\omega_{\mathbf{q}} 2 \pi \hbar \mathbf{p}} \cdot \varepsilon_{\mathbf{q}}^{\mu}$ the normalization and influence of the polarization $\varepsilon_{\mathbf{q}}^{\mu}$. The incoming electric field is characterized by a Gaussian beam propagating along the $z$ direction with width $w_{0}$ and polarization parallel to $\mathbf{p}$; the precise mode function is denoted as $u(\mathbf{r})$ and gives rise to the transverse mode area $A=\pi w_{0}^{2} / 2$. Therefore, this incoming state couples coherently to the $W$ state of the superatom,

$$
|W\rangle=\frac{1}{\sqrt{N}} \int d \mathbf{r} u(\mathbf{r}) S^{+}(\mathbf{r})|0\rangle .
$$

Here, $N$ denotes the relevant number of particles overlapping with the incoming mode of the photonic state, i.e.,

$$
N=\int d \mathbf{r}|u(\mathbf{r})|^{2}\left\langle 0\left|\psi_{g}^{\dagger}(\mathbf{r}) \psi_{g}(\mathbf{r})\right| 0\right\rangle .
$$

In general, this quantity varies within each shot of the experiment, as the position of atoms is randomly distributed, but its fluctuations are suppressed by $\Delta N / \bar{N} \sim 1 / \sqrt{\bar{N}}$ and can be safely ignored for $10^{4}$ particles participating in the superatom; here, $\bar{N}$ is the mean contributing atom number after averaging over many realizations. In the experimentally relevant regime with $\lambda \ll w_{0}, \sigma_{z}$ and $w_{0} \ll \sigma_{r}$, we obtain

$\bar{N}=\int d \mathbf{r}|u(\mathbf{r})|^{2} n(\mathbf{r})=\frac{(2 \pi)^{3 / 2}}{4} w_{0}^{2} \sigma_{z} n_{0}=(2 \pi)^{1 / 2} \sigma_{z} A n_{0}$.

In order to understand the collective enhancement of the decay of the superatom state $|W\rangle$, we determine its decay rate within Fermi's golden rule. The averaged decay rate into a photonic mode $\mathbf{q}$ with polarization $\varepsilon_{\mathbf{q}}^{\mu}$ takes the form 


$$
\begin{aligned}
\bar{\Gamma}_{\mathbf{q}, \mu} & =\frac{2 \pi g^{2}}{\hbar} \delta(\hbar \omega-\hbar c|\mathbf{q}|)\left|c_{\mathbf{q}}^{\mu}\right|^{2} \int d \mathbf{r}^{\prime} d \mathbf{r} e^{i \mathbf{q}\left(\mathbf{r}-\mathbf{r}^{\prime}\right)}\left\langle\frac{\psi_{g}^{\dagger}(\mathbf{r}) \psi_{g}(\mathbf{r}) \psi_{g}^{\dagger}\left(\mathbf{r}^{\prime}\right) \psi_{g}\left(\mathbf{r}^{\prime}\right)}{N}\right\rangle u^{*}\left(\mathbf{r}^{\prime}\right) u(\mathbf{r}) \\
& =\frac{2 \pi g^{2}}{\hbar} \delta(\hbar \omega-\hbar c|\mathbf{q}|)\left|c_{\mathbf{q}}^{\mu}\right|^{2}\left[1+\frac{1}{\bar{N}}\left|\int d \mathbf{r} e^{-i \mathbf{q r}} u(\mathbf{r}) n(\mathbf{r})\right|^{2}+O(\Delta N / \bar{N})\right] .
\end{aligned}
$$

Here, $\omega$ denotes the optical frequency of the transition. The first term gives rise to the standard spontaneous decay rate $\Gamma=4 g^{2} \omega^{3} / 3 \hbar c^{3}$ for a single atom. We therefore conclude that the superatom exhibits an incoherent decay process into an arbitrary photon mode $\mathbf{q}$, giving rise to the conventional spontaneous decay rate. In turn, the second term characterizes the possibility for collective enhancement of the decay into a specific mode. However, in the experimental parameter regime with $w\left(\sigma_{z}\right)<\sigma_{r}$, this collective decay only provides a significant contribution into the forward direction with an opening angle

$$
\sin ^{2} \theta \lesssim \frac{1}{\pi^{2}} \frac{\lambda^{2}}{w_{0}^{2}}
$$

These directions are, however, comparable to the angular spread of the Gaussian incoming beam. In particular, the back scattering is also suppressed by $\exp \left(-8 \pi^{2} \sigma_{z}^{2} / \lambda^{2}\right)$. Therefore, it is convenient to determine the spontaneous emission of the superatom state into the forwardpropagating Gaussian beam with mode $u(\mathbf{r})$ and polarization $\varepsilon^{\mu}=\mathbf{p}$, i.e.,

$$
\kappa=\frac{2 \pi g^{2}}{\hbar} \int \frac{d k}{2 \pi} \delta(\hbar \omega-\hbar c k) \frac{\left|c_{k}^{\mu}\right|^{2}}{A}\left[1+\left.\left.\frac{1}{\bar{N}}\left|\int d \mathbf{r}\right| u(\mathbf{r})\right|^{2} n(\mathbf{r})\right|^{2}\right]=\frac{2 \pi(\bar{N}+1) g^{2}}{A} \frac{\omega}{\hbar c} .
$$

Here, $A=\pi w_{0}^{2} / 2$ denotes the transverse mode volume of the Gaussian beam, and in the following discussion, we can well approximate $\bar{N}+1 \approx \bar{N}$. For a transverse width of the atomic density distribution $\sigma_{r} \lesssim w_{0}$, transitions into higher Gaussian modes are possible, and the determination of the decay rate into these modes is straightforward; these terms describe the fact that a narrow atomic medium in free space acts as a lens for the incoming photons.

We conclude, from this analysis, that the superatom state $|W\rangle$ collectively couples to a one-dimensional channel of forward-propagating modes. The latter are described by the Gaussian beam of the incoming probe field, and the collective coupling strength takes the form

$$
g_{\mathrm{col}}=2 \sqrt{\kappa}=\sqrt{\frac{8 \pi \bar{N} g^{2} \omega}{A \hbar c}}=\sqrt{\frac{3 \bar{N} \Gamma \lambda^{2}}{2 \pi A}} .
$$

The system therefore reduces to the Hamiltonian in Eq. (1) and the expression for the electric-field operator in Eq. (4). Note that, in addition, the superatom also exhibits an internal dynamics by the virtual exchange of photons [27,28], coupling the $|W\rangle$ state to the dark states. For the narrow bandwidth pulses used in the experiment, the variation in momentum of the electric field is small, and we can safely ignore any changes in the transverse wave function of the Gaussian beam. Furthermore, the incoherent spontaneous emission into the transverse channels, as well as back scattering, is well accounted for by the single-atom decay rate $\Gamma$.
In order to compare the two-level model presented in this appendix with the experimentally obtained results, we must consider the adiabatic elimination of the intermediate state present in the experiment. In this case, the effective coupling strength is $g_{\mathrm{col}}^{\text {eff }}=g_{\mathrm{col}} \Omega / 2 \Delta$ and, respectively, $\kappa^{\mathrm{eff}}=\left(g_{\mathrm{col}}^{\mathrm{eff}}\right)^{2} / 4=0.27 \mu \mathrm{s}^{-1}$.

[1] C. Cohen-Tannoudji, J. Dupont-Roc, and G. Grynberg, Photons and Atoms: Introduction to Quantum Electrodynamics (Wiley-VCH, New York, 1997).

[2] H. J. Kimble, The Quantum Internet, Nature (London) 453, 1023 (2008).

[3] A. Reiserer and G. Rempe, Cavity-Based Quantum Networks with Single Atoms and Optical Photons, Rev. Mod. Phys. 87, 1379 (2015).

[4] B. Hacker, S. Welte, G. Rempe, and S. Ritter, A PhotonPhoton Quantum Gate Based on a Single Atom in an Optical Resonator, Nature (London) 536, 193 (2016).

[5] I. Shomroni, S. Rosenblum, Y. Lovsky, O. Bechler, G. Guendelman, and B. Dayan, All-Optical Routing of Single Photons by a One-Atom Switch Controlled by a Single Photon, Science 345, 903 (2014).

[6] T. G. Tiecke, J. D. Thompson, N. P. de Leon, L. R. Liu, Vladan Vuletic, and M. D. Lukin, Nanophotonic Quantum Phase Switch with a Single Atom, Nature (London) 508, 241 (2014).

[7] B. W. Shore and P. L. Knight, The Jaynes-Cummings Model, J. Mod. Opt. 40, 1195 (1993). 
[8] J. M. Raimond, M. Brune, and S. Haroche, Manipulating Quantum Entanglement with Atoms and Photons in a Cavity, Rev. Mod. Phys. 73, 565 (2001).

[9] A. Wallraff, D. I. Schuster, A. Blais, L. Frunzio, R.-S. Huang, J. Majer, S. Kumar, S. M. Girvin, and R. J. Schoelkopf, Strong Coupling of a Single Photon to a Superconducting Qubit Using Circuit Quantum Electrodynamics, Nature (London) 431, 162 (2004).

[10] R. J. Schoelkopf and S. M. Girvin, Wiring up Quantum Systems, Nature (London) 451, 664 (2008).

[11] H. Pichler, T. Ramos, A. J. Daley, and P. Zoller, Quantum Optics of Chiral Spin Networks, Phys. Rev. A 91, 042116 (2015).

[12] A. N. Vamivakas, M. Atatüre, J. Dreiser, S. T. Yilmaz, A. Badolato, A. K. Swan, B. B. Goldberg, A. Imamoglu, and M. S. Ünlü, Strong Extinction of a Far-Field Laser Beam by a Single Quantum Dot, Nano Lett. 7, 2892 (2007).

[13] M. K. Tey, Z. Chen, S. A. Aljunid, B. Chng, F. Huber, G. Maslennikov, and C. Kurtsiefer, Strong Interaction between Light and a Single Trapped Atom without the Need for a Cavity, Nat. Phys. 4, 924 (2008).

[14] A. Maser, B. Gmeiner, T. Utikal, S. Götzinger, and V. Sandoghdar, Few-Photon Coherent Nonlinear Optics with a Single Molecule, Nat. Photonics 10, 450 (2016).

[15] A. F. van Loo, A. Fedorov, K. Lalumière, B. C. Sanders, A. Blais, and A. Wallraff, Photon-Mediated Interactions between Distant Artificial Atoms, Science 342, 1494 (2013).

[16] J. Petersen, J. Volz, and A. Rauschenbeutel, Chiral Nanophotonic Waveguide Interface Based on Spin-Orbit Interaction of Light, Science 346, 67 (2014).

[17] S. Faez, P. Türschmann, H. R. Haakh, S. Götzinger, and V. Sandoghdar, Coherent Interaction of Light and Single Molecules in a Dielectric Nanoguide, Phys. Rev. Lett. 113, 213601 (2014).

[18] P. Lodahl, S. Mahmoodian, and S. Stobbe, Interfacing Single Photons and Single Quantum Dots with Photonic Nanostructures, Rev. Mod. Phys. 87, 347 (2015).

[19] A. Goban, C.-L. Hung, J. D. Hood, S.-P. Yu, J. A. Muniz, O. Painter, and H.J. Kimble, Superradiance for Atoms Trapped Along a Photonic Crystal Waveguide, Phys. Rev. Lett. 115, 063601 (2015).

[20] A. Sipahigil, R. E. Evans, D. D. Sukachev, M. J. Burek, J. Borregaard, M. K. Bhaskar, C. T. Nguyen, J. L. Pacheco, H. A. Atikian, C. Meuwly et al., An Integrated Diamond Nanophotonics Platform for Quantum-Optical Networks, Science 354, 847 (2016).

[21] M. K. Bhaskar, D. D. Sukachev, A. Sipahigil, R. E. Evans, M. J. Burek, C. T. Nguyen, L. J. Rogers, P. Siyushev, M. H. Metsch et al., Quantum Nonlinear Optics with a Germanium-Vacancy Color Center in a Nanoscale Diamond Waveguide, Phys. Rev. Lett. 118, 223603 (2017).

[22] M. D. Lukin, M. Fleischhauer, R. Cote, L. M. Duan, D. Jaksch, J. I. Cirac, and P. Zoller, Dipole Blockade and Quantum Information Processing in Mesoscopic Atomic Ensembles, Phys. Rev. Lett. 87, 037901 (2001).

[23] E. Urban, T. A. Johnson, T. Henage, L. Isenhower, D. D. Yavuz, T. G. Walker, and M. Saffman, Observation of Rydberg Blockade between Two Atoms, Nat. Phys. 5, 110 (2009).
[24] A. Gaëtan, Y. Miroshnychenko, T. Wilk, A. Chotia, M. Viteau, D. Comparat, P. Pillet, A. Browaeys, and P. Grangier, Observation of Collective Excitation of Two Individual Atoms in the Rydberg Blockade Regime, Nat. Phys. 5, 115 (2009).

[25] Y. O. Dudin, L. Li, F. Bariani, and A. Kuzmich, Observation of Coherent Many-Body Rabi Oscillations, Nat. Phys. 8, 790 (2012).

[26] M. O. Scully, E. S. Fry, C. H. Raymond Ooi, and K. Wódkiewicz, Directed Spontaneous Emission from an Extended Ensemble of $N$ Atoms: Timing Is Everything, Phys. Rev. Lett. 96, 010501 (2006).

[27] R. H. Lehmberg, Radiation from an N-Atom System. I. General Formalism, Phys. Rev. A 2, 883 (1970).

[28] A. A. Svidzinsky, J.-T. Chang, and M. O. Scully, Dynamical Evolution of Correlated Spontaneous Emission of a Single Photon from a Uniformly Excited Cloud of n Atoms, Phys. Rev. Lett. 100, 160504 (2008).

[29] D. Porras and J. I. Cirac, Collective Generation of Quantum States of Light by Entangled Atoms, Phys. Rev. A 78, 053816 (2008).

[30] Y. Miroshnychenko, U. V. Poulsen, and K. Mølmer, Directional Emission of Single Photons from Small Atomic Samples, Phys. Rev. A 87, 023821 (2013).

[31] M. O. Araújo, I. Krešić, R. Kaiser, and W. Guerin, Superradiance in a Large and Dilute Cloud of Cold Atoms in the Linear-Optics Regime, Phys. Rev. Lett. 117, 073002 (2016).

[32] S. J. Roof, K. J. Kemp, M. D. Havey, and I. M. Sokolov, Observation of Single-Photon Superradiance and the Cooperative Lamb Shift in an Extended Sample of Cold Atoms, Phys. Rev. Lett. 117, 073003 (2016).

[33] J. Honer, R Löw, H. Weimer, T. Pfau, and H. P. Büchler, Artificial Atoms Can Do More Than Atoms: Deterministic Single Photon Subtraction from Arbitrary Light Fields, Phys. Rev. Lett. 107, 093601 (2011).

[34] C. Tresp, C. Zimmer, I. Mirgorodskiy, H. Gorniaczyk, A. Paris-Mandoki, and S. Hofferberth, Single-Photon Absorber Based on Strongly Interacting Rydberg Atoms, Phys. Rev. Lett. 117, 223001 (2016).

[35] T. M. Weber, M. Höning, T. Niederprüm, T. Manthey, O. Thomas, V. Guarrera, M. Fleischhauer, G. Barontini, and H. Ott, Mesoscopic Rydberg-Blockaded Ensembles in the Superatom Regime and Beyond, Nat. Phys. 11, 157 (2015).

[36] J. Zeiher, P. Schauß, S. Hild, T. Macrì, I. Bloch, and C. Gross, Microscopic Characterization of Scalable Coherent Rydberg Superatoms, Phys. Rev. X 5, 031015 (2015).

[37] H. Labuhn, D. Barredo, S. Ravets, S. de Léséleuc, T. Macrì, T. Lahaye, and A. Browaeys, Tunable Two-Dimensional Arrays of Single Rydberg Atoms for Realizing Quantum Ising Models, Nature (London) 534, 667 (2016).

[38] T. Shi, D. E. Chang, and J. I. Cirac, Multiphoton-Scattering Theory and Generalized Master Equations, Phys. Rev. A 92, 053834 (2015).

[39] G. Leuchs and M. Sondermann, Light-Matter Interaction in Free Space, J. Mod. Opt. 60, 36 (2013).

[40] U. Schnorrberger, J. D. Thompson, S. Trotzky, R. Pugatch, N. Davidson, S. Kuhr, and I. Bloch, Electromagnetically Induced Transparency and Light Storage in an Atomic Mott Insulator, Phys. Rev. Lett. 103, 033003 (2009). 
[41] Y. O. Dudin, L. Li, and A. Kuzmich, Light Storage on the Time Scale of a Minute, Phys. Rev. A 87, 031801 (2013).

[42] Y. Wang, J. ří Minář, L. Sheridan, and V. Scarani, Efficient Excitation of a Two-Level Atom by a Single Photon in a Propagating Mode, Phys. Rev. A 83, 063842 (2011).

[43] V. Leong, M. A. Seidler, M. Steiner, A. Cerè, and C. Kurtsiefer, Time-Resolved Scattering of a Single Photon by a Single Atom, Nat. Commun. 7, 13716 (2016).

[44] M. Lax, Quantum Noise. XI. Multitime Correspondence between Quantum and Classical Stochastic Processes, Phys. Rev. 172, 350 (1968).

[45] V. I. Rupasov and V. I. Yudson, Exact Dicke Superradiance Theory: Bethe Wavefunctions in the Discrete Atom Model, Sov. Phys. JETP 59, 478 (1984).

[46] V. I. Yudson, Dynamics of Integrable Quantum Systems, Sov. Phys. JETP 61, 1043 (1985).

[47] V. I. Yudson and P. Reineker, Multiphoton Scattering in a One-Dimensional Waveguide with Resonant Atoms, Phys. Rev. A 78, 052713 (2008).
[48] E. Rephaeli and S. Fan, Stimulated Emission from a Single Excited Atom in a Waveguide, Phys. Rev. Lett. 108, 143602 (2012).

[49] K. Stannigel, P. Rabl, and P. Zoller, Driven-Dissipative Preparation of Entangled States in Cascaded QuantumOptical Networks, New J. Phys. 14, 063014 (2012).

[50] P. Lodahl, S. Mahmoodian, S. Stobbe, A. Rauschenbeutel, P. Schneeweiss, J. Volz, H. Pichler, and P. Zoller, Chiral Quantum Optics, Nature (London) 541, 473 (2017).

[51] C. Gardiner and P. Zoller, Quantum Noise: A Handbook of Markovian and Non-Markovian Quantum Stochastic Methods with Applications to Quantum Optics, Springer Series in Synergetics (Springer, New York, 2004).

[52] B. R. Mollow, Pure-State Analysis of Resonant Light Scattering: Radiative Damping, Saturation, and Multiphoton Effects, Phys. Rev. A 12, 1919 (1975).

[53] T. Caneva, M. T. Manzoni, T. Shi, J. S. Douglas, J. Ignacio Cirac, and D. E. Chang, Quantum Dynamics of Propagating Photons with Strong Interactions: A Generalized InputOutput Formalism, New J. Phys. 17, 113001 (2015). 